\title{
PRONY ESTIMATION OF AR PARAMETERS OF AN ARMA TIME SERIES
}

\author{
S. Hu AND S. M. WU \\ Department of Mechanical Engineering and Applied Mechanics, University of Michigan, \\ Ann Arbor, Michigan 48109, U.S.A.
}

(Received July 1988, accepted September 1988)

\begin{abstract}
The auto-covariance function of a white noise excited time series can be decomposed into the contributions of different modes, therefore having the same structure as that of the impulse response of a deterministic system. By matching the auto-covariance of the data with that of the ARMA model, the estimation of the characteristic roots of the system and the dispersion coefficients can be implemented using the Prony method, therefore the estimation of the AR parameters becomes a linear least squares problem. It is found that this estimate for the AR parameters of an ARMA model is identical to the asymptotically unbiased estimate using modified Yule-Walker equation for ARMA $(n, n-1)$.
\end{abstract}

\section{INTRODUCTION}

The mixed autoregressive moving average (ARMA) model can be used to represent the dynamics of a mechanical system. It was shown by Bartlett [1] that the sampling of a continuous AR process of order $n$ results in a discrete ARMA process of order $n, n-1$, i.e. ARMA $(n, n-1)$. The example originally examined was the dynamics of a Brownian motion excited pendulum using discrete observations of the continuous system. Bartlett showed that the discrete time series that is covariance equivalent to the system

$$
\frac{\mathrm{d}^{2} x(t)}{\mathrm{d} t^{2}}+2 \zeta \omega_{n} \frac{\mathrm{d} x(t)}{\mathrm{d} t}+\omega_{n}^{2}=a(t)
$$

is

$$
x_{t}=\phi_{1} x_{t-1}+\phi_{2} x_{t-2}+a_{t}-\theta_{1} a_{t-1}
$$

where $a_{t}$ is a zero mean uncorrelated series. $\phi_{i}$ 's are called the autoregressive (AR) parameters, and $\theta_{i}$ 's the moving average (MA) parameter.

Generally, an ARMA $(n, m)$ model is of the form

$$
x_{t}=\phi_{1} x_{t-1}+\phi_{2} x_{t-2+}+\cdots+\phi_{n} x_{t-n}+a_{t}-\theta_{1} a_{t-1}-\cdots-\theta_{m} a_{t-m}
$$

\subsection{REPRESENTATION OF DYNAMICS}

For the ARMA $(n, m)$ model given in equation (1), its theoretical auto-covariance function can be expressed as [2]

$$
\gamma_{k}=d_{1} \lambda_{1}^{k}+d_{2} \lambda_{2}^{k}+\cdots+d_{n} \lambda_{n}^{k}
$$

where $\lambda_{i}$ 's are the characteristic roots of

$$
1-\phi_{1} B-\cdots-\phi_{n} B^{n}=0
$$


or

$$
\left(1-\lambda_{1} B\right)\left(1-\lambda_{2} B\right) \cdots\left(1-\lambda_{n} B\right)=0
$$

and the $d_{i}$ represents the contribution of the $i$ th dynamic mode of the system $\lambda_{i}$ to the variance $\gamma_{0}$, and $d_{i} / \gamma_{0} \times 100 \%$ is called the dispersion coefficient.

The estimate for the auto-covariance function is given by

$$
\hat{\gamma}_{k}=\frac{1}{N} \sum_{t=k+1}^{N}\left(x_{t}-\bar{x}\right)\left(x_{t-k}-\bar{x}\right)
$$

where

$$
\bar{x}=\frac{1}{N} \sum_{i=1}^{N} x_{i}
$$

and $N$ is the total number of data.

There are, of course, other methods of estimating the auto-covariance, but the estimator equation (5) has the attractive properties that its mean squared error is generally smaller than that of other estimators [3].

\subsection{MODIFIED YULE-WALKER EQUATION}

The estimation of the parameters of the ARMA model has been an active field in recent years. The maximum likelihood method provides good estimate for parameters of the ARMA model [4]. However, because of the severe non-linearity and local minimum problem, many sub-optimal estimation procedures have been suggested [5].

Gersch [6] showed that the modified Yule-Walker equation provides the asymptotically unbiased estimate for the AR parameters of an ARMA model. The estimator for the AR parameters of ARMA $(n, m)$ model using modified Yule-Walker equation is given by

$$
\left[\begin{array}{cccc}
\hat{\gamma}_{m} & \hat{\gamma}_{m-1} & \cdots & \hat{\gamma}_{m-n+1} \\
\hat{\gamma}_{m+1} & \hat{\gamma}_{m} & \cdots & \hat{\gamma}_{m-n+2} \\
\vdots & & & \\
\hat{\gamma}_{m+n-1} & \hat{\gamma}_{m+n-2} & \cdots & \hat{\gamma}_{m}
\end{array}\right]\left\{\begin{array}{c}
\phi_{1} \\
\phi_{2} \\
\vdots \\
\phi_{n}
\end{array}\right\}=\left\{\begin{array}{c}
\hat{\gamma}_{m+1} \\
\hat{\gamma}_{m+2} \\
\vdots \\
\hat{\gamma}_{m+n}
\end{array}\right\} .
$$

\subsection{THE PRONY METHOD}

The Prony method [7], a technique for modeling data of equally spaced samples by a linear combination of exponentials assumes the discrete time function of the form

$$
z_{k}=\sum_{j=1}^{n} b_{j} z_{j}^{k}
$$

for $k=0,1, \ldots, n-1$. It has found most of its application in transient analysis, such as finding resonant modes in experimental modal analysis. It has also been used to perform harmonic decomposition [5]. However, it has not been used for direct parameter estimation of an ARMA model.

In this paper, we develop the estimation for the AR parameters of an ARMA model using the Prony method, and show that the resulting estimate of the AR parameters are identical to that from modified Yule-Walker equation for ARMA $(n, n-1)$ model. 


\section{THE ESTIMATION PROBLEM}

The model equation (7) assumed by the Prony method has the same structure as that of the auto-covariance function of the $\operatorname{ARMA}(n, m)$ model shown in equation (2). Equating the auto-covariance of the data at different lag with that of the model, we obtain the following system of equations:

$$
\left.\begin{array}{rl}
\hat{\gamma}_{0} & =d_{1}+d_{2}+\cdots+d_{n} \\
\hat{\gamma}_{1} & =d_{1} \lambda_{1}+d_{2} \lambda_{2}+\cdots+d_{n} \lambda_{n} \\
\vdots & \\
\hat{\gamma}_{2 n-1} & =d_{1} \lambda_{1}^{2 n-1}+d_{2} \lambda_{2}^{2 n-1}+\cdots+d_{n} \lambda_{n}^{2 n-1}
\end{array}\right\} .
$$

Multiplying both sides of the first equation of (8) by $\beta_{0}$, the second equation of (8) by $\beta_{1}, \ldots$, and the $n+1$ th equation of $(8)$ by $\beta_{n}$, we have

$$
\left.\begin{array}{c}
\beta_{0} \hat{\gamma}_{0}=\beta_{0} d_{1}+\beta_{0} d_{2}+\cdots+\beta_{0} d_{n} \\
\beta_{1} \hat{\gamma}_{1}=\beta_{1} d_{1} \lambda_{1}+\beta_{1} d_{2} \lambda_{2}+\cdots+\beta_{1} d_{n} \lambda_{n} \\
\vdots \\
\beta_{n} \hat{\gamma}_{n}=\beta_{n} d_{1} \lambda_{1}^{n}+\beta_{n} d_{2} \lambda_{2}^{n}+\cdots+\beta_{n} d_{n} \lambda_{n}^{n}
\end{array}\right\} .
$$

Adding up the equations, we have

$$
\sum_{j=0}^{n} \beta_{j} \hat{\gamma}_{j}=\sum_{i=1}^{n} d_{i}\left(\sum_{j=0}^{n} \beta_{j} \lambda_{i}^{j}\right) .
$$

The $\beta_{i}$ 's can be chosen as the coefficients of the following equation

$$
\beta_{0}+\beta_{1} \lambda+\beta_{2} \lambda^{2}+\cdots+\beta_{n} \lambda^{n}=0
$$

with $\beta_{n}=1$ and the roots $\lambda_{1}, \ldots, \lambda_{n}$.

The right-hand side of equation (10) is zero for any $\lambda_{i}$, and then equation (10) becomes

$$
\sum_{j=0}^{n} \beta_{j} \hat{\gamma}_{j}=0
$$

i.e.

$$
\beta_{0} \hat{\gamma}_{0}+\beta_{1} \hat{\gamma}_{1}+\cdots+\beta_{n-1} \hat{\gamma}_{n-1}=-\hat{\gamma}_{n}
$$

Repeating the process from equation (9) to equation (12) in a similar way, multiplying both sides of the second equation of $(8)$ by $\beta_{0}$, the third equation of $(8)$ by $\beta_{1}, \ldots$, and the $n+2$ th equation of $(8)$ by $\beta_{n}$, we have,

$$
\beta_{0} \hat{\gamma}_{1}+\beta_{1} \hat{\gamma}_{2}+\cdots+\beta_{n-1} \hat{\gamma}_{n}=-\hat{\gamma}_{n+1} \text {. }
$$

After repeating the above process $n$ times, we have the following system of equations

$$
\left.\begin{array}{c}
\beta_{0} \hat{\gamma}_{0}+\beta_{1} \hat{\gamma}_{1}+\cdots+\beta_{n-1} \hat{\gamma}_{n-1}=-\hat{\gamma}_{n} \\
\beta_{0} \hat{\gamma}_{1}+\beta_{1} \hat{\gamma}_{2}+\cdots+\beta_{n-1} \hat{\gamma}_{n}=-\hat{\gamma}_{n+1} \\
\vdots \\
\beta_{0} \hat{\gamma}_{n-1}+\beta_{1} \hat{\gamma}_{n}+\cdots+\beta_{n-1} \hat{\gamma}_{2 n-2}=-\hat{\gamma}_{2 n-1}
\end{array}\right\} .
$$

This system of equations can be solved using a linear least squares routine.

$$
\hat{\Theta}=\left(X^{T} X\right)^{-1}\left(X^{T} Y\right)
$$


where "T" denotes the transpose of a matrix,

$$
\begin{gathered}
X=\left[\begin{array}{cccc}
\hat{\gamma}_{0} & \hat{\gamma}_{1} & \cdots & \hat{\gamma}_{n-1} \\
\hat{\gamma}_{1} & \hat{\gamma}_{2} & \cdots & \hat{\gamma}_{n} \\
\vdots & & & \\
\hat{\gamma}_{n-1} & \hat{\gamma}_{n} & \cdots & \hat{\gamma}_{2 n-2}
\end{array}\right] \\
Y=\left\{\begin{array}{c}
-\hat{\gamma}_{n} \\
-\hat{\gamma}_{n+1} \\
\vdots \\
-\hat{\gamma}_{2 n-1}
\end{array}\right\}
\end{gathered}
$$

and $\Theta$ is the parameter vector,

$$
\Theta=\left\{\begin{array}{c}
\beta_{0} \\
\beta_{1} \\
\vdots \\
\beta_{n-1}
\end{array}\right\}
$$

After identifying the parameters $\beta_{i}$ 's, the characteristic root $\lambda_{i}$ 's of the system can be calculated by solving the polynomial equation (11).

$$
\beta_{0}+\beta_{1} \lambda+\beta_{2} \lambda^{2}+\cdots+\beta_{n-1} \lambda^{n-1}+\lambda^{n}=0 .
$$

From equation (8), we have

$$
\left[\begin{array}{cccc}
1 & 1 & \cdots & 1 \\
\lambda_{1} & \lambda_{2} & \cdots & \lambda_{n} \\
\vdots & & & \\
\lambda_{1}^{n-1} & \lambda_{2}^{n-1} & \cdots & \lambda_{n}^{n-1}
\end{array}\right]\left\{\begin{array}{c}
d_{2} \\
d_{2} \\
\vdots \\
d_{n}
\end{array}\right\}=\left\{\begin{array}{c}
\hat{\gamma}_{0} \\
\hat{\gamma}_{1} \\
\vdots \\
\hat{\gamma}_{n-1}
\end{array}\right\}
$$

Again, the above equation can be solved for $d_{i}$ 's using a linear least squares routine.

2.1. THE MEANING OF $\boldsymbol{\beta}_{i}$

For an $\operatorname{ARMA}(n, m)$ model, the characteristic equation becomes

$$
1-\phi_{1} B-\phi_{2} B^{2}-\cdots-\phi_{n} B^{n}=0
$$

or

$$
\lambda_{i}^{n}-\phi_{1} \lambda_{i}^{n-1}-\cdots-\phi_{n-1} \lambda_{i}-\phi_{n}=0 .
$$

Compare equations (17) and (11), we have,

$$
\left.\begin{array}{c}
\phi_{1}=-\beta_{n-1} \\
\phi_{2}=-\beta_{n-2} \\
\vdots \\
\phi_{n}=-\beta_{0}
\end{array}\right\}
$$

That is, the least square estimate from equation (14) is directly related to the AR parameters of the ARMA model. The estimation of the AR parameters of the ARMA model becomes a linear least square problem. 
Rewrite equation (13) using $\phi_{i}$ 's instead of $\beta_{i}$ 's, we have

$$
\left[\begin{array}{cccc}
\hat{\gamma}_{n-1} & \hat{\gamma}_{n-2} & \cdots & \hat{\gamma}_{0} \\
\hat{\gamma}_{n} & \hat{\gamma}_{n-1} & \cdots & \hat{\gamma}_{1} \\
\vdots & & & \\
\hat{\gamma}_{2 n-2} & \hat{\gamma}_{2 n-3} & \cdots & \hat{\gamma}_{n-1}
\end{array}\right]\left\{\begin{array}{c}
\phi_{1} \\
\phi_{2} \\
\vdots \\
\phi_{n}
\end{array}\right\}=\left\{\begin{array}{c}
\hat{\gamma} \\
\hat{\gamma}_{n+1} \\
\vdots \\
\hat{\gamma}_{2 n-1}
\end{array}\right\} .
$$

Comparing equation (6) with equation (19), we can see that equation (19) is identical to the asymptotically unbiased estimate using the modified Yule-Walker equation for the AR parameters of an ARMA $(n, n-1)$ model.

\section{CONCLUSIONS}

This paper provides another interpretation for the modified Yule-Walker estimation for the AR parameters of the ARMA model-Prony estimation using autocovariance function. The auto-covariance function of an autoregressive moving average time series is decomposed into the contributions of different modes. The estimation of the AR parameters of an ARMA model can be implemented using the Prony method, and this estimate for the AR parameters is identical to the asymptotically unbiased estimate of the AR parameters of an ARMA $(n, n-1)$ model using modified Yule-Walker equation.

\section{REFERENCES}

1. M. S. BARTLETT 1946 Journal of the Royal Statistical Society B8, 27-41. On the theoretical specification and sampling properties of autocorrelated time series.

2. S. M. PANDIT and S. M. WU 1983 Time Series and System Analysis with Applications, pp. 111-130. New York: John Wiley.

3. G. M. JENKINS and D. G. WATts 1969 Spectral Analysis and Its Applications, pp. 171-241. San Francisco: Holden-Day.

4. K. J. ASTROM and P. EYKHOFF 1971 Automatica 7, 123-162. System identification-a survey.

5. S. M. KAY 1981 Proceedings of the IEEE 69, 1380-1419. Spectral analysis-a modern perspective.

6. W. GERSCH 1970 IEEE, Transactions on Automotive Control 15, 583-588. Estimation of the autoregressive parameters of a mixed autoregressive moving average time series.

7. P. DAVIES 1983 Journal of Sound and Vibration 89, 571-583. A recursive approach to Prony parameter estimation. 